\title{
Influence of Disease Duration on Circulating Levels of miRNAs in Children and Adolescents with New Onset Type 1 Diabetes
}

\author{
Nasim Samandari ${ }^{1}$, Aashiq H. Mirza ${ }^{1}$, Simranjeet Kaur ${ }^{1,2}{ }^{1}$, Philip Hougaard ${ }^{3}$, \\ Lotte B. Nielsen ${ }^{1}$, Siri Fredheim ${ }^{1}$, Henrik B. Mortensen ${ }^{1,4}$ and Flemming Pociot $1,2,4, *$ (D) \\ 1 Copenhagen Diabetes Research Centre (CPH-DIRECT), Department of Paediatrics, Herlev and Gentofte \\ Hospitals, Herlev Ringvej 75, 2730 Herlev, Denmark; nasim.samandari@regionh.dk (N.S.); \\ aah2003@med.cornell.edu (A.H.M.); simranjeet.kaur@regionh.dk (S.K.); lottebn2@gmail.com (L.B.N.); \\ sirifredheim@dadlnet.dk (S.F.); henrik.bindesboel.mortensen@regionh.dk (H.B.M.) \\ 2 Steno Diabetes Center Copenhagen, 2820 Gentofte, Denmark \\ 3 Research Unit of Epidemiology, Biostatistics and Biodemography, University of Southern Denmark, \\ 5230 Odense M, Denmark; phhougaard@mail.dk \\ 4 Faculty of Health and Medical Sciences, University of Copenhagen, 2200 Copenhagen N, Denmark \\ * Correspondence: flemming.pociot@regionh.dk; Tel.: +45-3-968-0800
}

Received: 4 October 2018; Accepted: 19 November 2018; Published: 21 November 2018

\begin{abstract}
Circulating microRNAs (miRNAs) have been implicated in several pathologies including type 1 diabetes. In the present study, we aimed to identify circulating miRNAs affected by disease duration in children with recent onset type 1 diabetes. Forty children and adolescents from the Danish Remission Phase Cohort were followed with blood samples drawn at 1, 3, 6, 12, and 60 months after diagnosis. Pancreatic autoantibodies were measured at each visit. Cytokines were measured only the first year. miRNA expression profiling was performed by RT-qPCR. The effect of disease duration was analyzed by mixed models for repeated measurements adjusted for sex and age. Eight miRNAs (hsa-miR-10b-5p, hsa-miR-17-5p, hsa-miR-30e-5p, hsa-miR-93-5p, hsa-miR-99a-5p, hsa-miR-125b-5p, hsa-miR-423-3p, and hsa-miR-497-5p) were found to significantly change in expression (adjusted $p$-value $<0.05$ ) with disease progression. Three pancreatic autoantibodies, ICA, IA-2A, and GAD65A, and four cytokines, IL-4, IL-10, IL-21, and IL-22, were associated with the miRNAs at different time points. Pathway analysis revealed associations with various immune-mediated signaling pathways. Eight miRNAs that were involved in immunological pathways changed expression levels during the first five years after diagnosis and were associated with variations in cytokine and pancreatic antibodies, suggesting a possible effect on the immunological processes in the early phase of the disease.
\end{abstract}

Keywords: children; immunology; miRNA; partial remission phase; type 1 diabetes

\section{Introduction}

There is an increased interest in epigenetic mechanisms to understand disease pathogenesis and progression. Epigenetics involves the heritable modifications of gene expression without changes in the genome sequence, leading to an altered, sometimes unfavorable, phenotype, e.g., resulting in metabolic diseases such as diabetes. While it is recognized that a genetic component is essential to the risk of developing type 1 diabetes (T1D), data from twin studies suggests that genetics alone cannot explain the disease development [1,2]. Furthermore, the increasing incidence of T1D in younger children is at a rate which is too fast to be only accounted for by a genetic change. Thus, the role of other pathogenic influences, such as diet, environment, viral infections, interferons, and epigenetic modifications, has been suggested to contribute to the development of autoimmune diabetes [3-7]. 
MicroRNAs (miRNAs) are small non-coding RNAs with potent post-transcriptional regulatory properties which have shown potential as biomarkers for different pathophysiologies [8]. During recent years, the promising features of miRNAs as biomarkers associated with insulin production, residual $\beta$-cell function, and disease complications of diabetes have been investigated [9-11]. The knowledge emerging on the role of epigenetic control, such as miRNAs, DNA methylation patterns, and histone modifications, indicates that these regulatory mechanisms have an important function in T1D [12-14].

An important stage in the natural history of T1D is the spontaneous remission phase, which can be either complete, with temporary insulin independence, or partial. During this phase, which can last from weeks to several months, a decreased requirement of exogenous insulin is experienced and an improved metabolic control is observed [15]. The partial remission (PR) phase is a potential temporal opening for investigating the important immunoregulatory events taking place when the remaining $\beta$-cells are still able to produce enough insulin. Currently, the strategies employed so far have not yet been able to unravel the underlying mechanisms during this phase, making the development of future immunotherapies with the capabilities to extend the PR challenging.

Previously, we identified six miRNAs predictive for residual $\beta$-cell function and metabolic control in children newly diagnosed with T1D [11]. In the present study, we hypothesized that circulating miRNAs reflect the ongoing autoimmune process during the first year after disease onset. Thus, the objective of this study was to identify circulating miRNAs, which hold the potential to serve as biomarkers for the autoimmune and immunological status in newly diagnosed children and adolescents with T1D.

\section{Results}

\subsection{Technical Controls Prior to miRNA Profiling}

The demographic and anthropometric data characterizing the study cohort is presented in Table 1 and has also recently been described elsewhere [11]. Plasma samples from a subset of children and adolescents $(n=40)$ from this cohort collected at different time-points $(1,3,6,12$, and 60 months) after diagnosis were subjected to miRNA profiling. The steady expression levels of the RNA spike-ins, added in the RNA isolation and complementary DNA (cDNA) synthesis steps, indicated that the processes of RNA extraction, reverse transcription, and qPCR were efficient, and no inhibitors were present in the samples. Hemolysis of the samples varied between 5 and -2 , thus indicating that the samples were not affected by hemolysis

Table 1. Characteristics of 40 children and adolescents (study population) from the Danish Remission Phase Cohort and the entire cohort, respectively.

\begin{tabular}{cccccc}
\hline Study Population & 1 Month & 3 Months & 6 Months & 12 Months & 60 Months \\
\hline$n$ (girls/ $n$ ) & $40(24)$ & $37(22)$ & $33(19)$ & $34(20)$ & $38(23)$ \\
Age at diagnosis (years) & $8.7(3.4)$ & - & - & - & - \\
HbA1c (DCCT, \%) & $9.1(1.3)$ & $7.3(1.1)$ & $7.8(1.6)$ & $7.9(1.1)$ & $8.4(1.0)$ \\
HbA1c (IFCC,mmol/mol) & $76(14.2)$ & $56(12.0)$ & $62(17.5)$ & $63(12.0)$ & $68(11.0)$ \\
C-peptide (pmol/L) & $629(371)$ & $528(323)$ & $436(333)$ & $257(251)$ & $62(76)$ \\
IDAA1c & $10.8(2.0)$ & $9.2(1.5)$ & $10.1(2.2)$ & $11.0(1.8)$ & $12.0(1.9)$ \\
Insulin dose (units/kg/24h) & $0.45(0.27)$ & $0.47(0.23)$ & $0.58(0.26)$ & $0.78(0.27)$ & $0.88(0.32)$ \\
Autoantibody positivity (\%) & & & & & \\
GAD65A & 55 & 62 & 61 & 56 & 55 \\
IA & 80 & 100 & 100 & 100 & 78 \\
IA-2A & 73 & 68 & 70 & 68 & 74 \\
ICA & 93 & 92 & 94 & 94 & 92 \\
ZnT8tripleAb & 65 & 70 & 75 & 76 & 26 \\
\hline
\end{tabular}

Data are presented as means (SD), unless otherwise stated. SCP: stimulated C-peptide. IDAA1c $=$ HbA1c (percent) $+[4 \times$ insulin dose (units per kilogram per $24 \mathrm{~h}$ )]. GAD65A: autoantibodies against glutamic acid decarboxylase (GAD). IA: insulin autoantibodies. IA-2A: islet antigen 2 antibody. ICA: islet-cell antibody. ZnT8tribleAb: Zinc transporter 8 triple mix antibody variant containing the isotypes glutamine, arginine, and tryptophan. Missing data are either due to no visit, missing or lost sample, or drop-out. 


\section{2. miRNA Quantification in 182 Plasma Samples}

A selected panel of 179 human serum/plasma miRNAs was analyzed over the five time points after diagnosis. An average of 163 miRNAs were quantified and 100 were quantified in all samples.

\subsection{Effect of Disease Duration on miRNA Expression Levels during the First Five Years after Diagnosis}

Analysis by mixed model for repeated measurements indicated that 28 miRNAs varied in expression levels during the first five years after diagnosis $(p<0.05)$. After correcting for multiple testing, eight miRNAs (hsa-miR-10b-5p, hsa-miR-17-5p, hsa-miR-30e-5p, hsa-miR-93-5p, hsa-miR-99a-5p, hsa-miR-125b-5p, hsa-miR-423-3p, and hsa-miR-497-5p) showed variations in expression levels during the first five years after disease onset $(p<0.05)$ (Table 2). The results of these eight candidate miRNAs were adjusted for age and sex. After adjustments, the data revealed that age only had an effect on hsa-miR-423-3p ( $p=0.0088$, data not shown). Neither HbA1c nor stimulated C-peptide (SCP) correlated with any of the quantified miRNAs. The average expression levels of the eight candidate miRNAs with altered expression levels during the first five years are shown as a heat map in Figure 1.

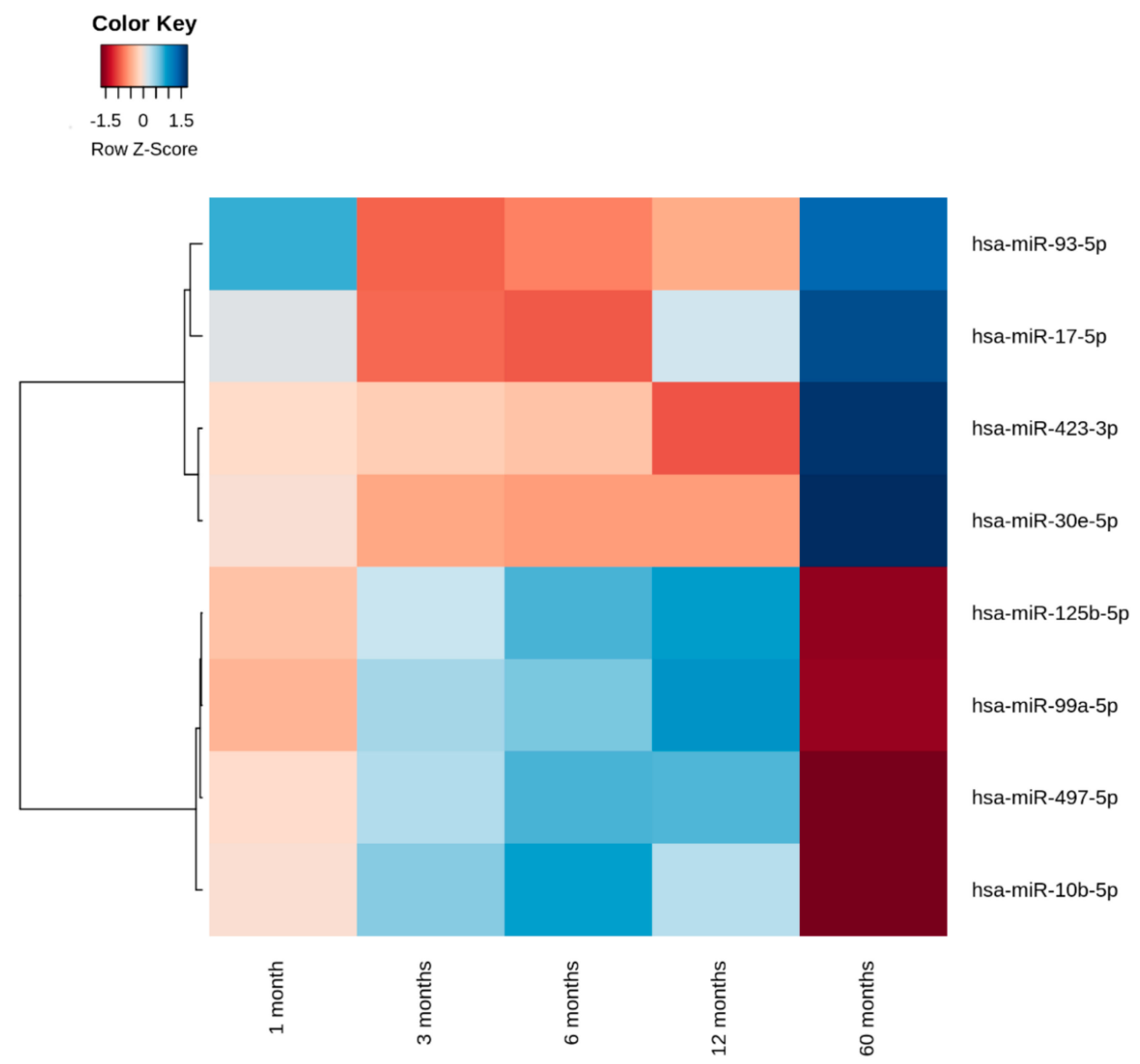

Figure 1. The eight candidate microRNAs (miRNAs) with changing expression levels during the first five years after diagnosis. The normalized expression $(\Delta \mathrm{Cp})$ values of the miRNAs at different time-points are clustered based on the distance measure (which is calculated as 1-cor) and transformed into Z-scores. cor = Pearson's correlation coefficient. 
Table 2. Effect of disease duration on miRNA expression level.

\begin{tabular}{ccc}
\hline miRNA & Unadjusted $p$-Value & Adjusted $p$-Value \\
\hline hsa-miR-99a-5p & $<0.00001$ & 0.00022 \\
hsa-miR-30e-5p & $<0.00001$ & 0.00027 \\
hsa-miR-497-5p & $<0.00001$ & 0.00041 \\
hsa-miR-10b-5p & 0.00001 & 0.00072 \\
hsa-miR-423-3p & 0.00001 & 0.00131 \\
hsa-miR-125b-5p & 0.00003 & 0.00252 \\
hsa-miR-17-5p & 0.00011 & 0.01057 \\
hsa-miR-93-5p & 0.00012 & 0.01094 \\
hsa-miR-146a-5p & 0.00094 & 0.08678 \\
hsa-miR-484 & 0.00195 & 0.17716 \\
hsa-miR-185-5p & 0.00208 & 0.18726 \\
hsa-miR-24-3 & 0.00291 & 0.25943 \\
hsa-miR-660-5p & 0.00944 & 0.83059 \\
hsa-miR-25-3p & 0.01088 & 0.94673 \\
hsa-let-7b-5p & 0.01133 & 0.97420 \\
hsa-miR-320a & 0.01236 & 0.98546 \\
hsa-miR-223-3p & 0.01248 & 0.98546 \\
hsa-let-7g-5p & 0.01553 & 0.98546 \\
hsa-miR-20a-5p & 0.01815 & 0.98546 \\
hsa-let-7i-5p & 0.02298 & 0.98546 \\
hsa-miR-142-3p & 0.02412 & 0.98546 \\
hsa-miR-32-5p & 0.02544 & 0.98546 \\
hsa-miR-486-5p & 0.02989 & 0.98546 \\
hsa-miR-142-5p & 0.04400 & 0.98546 \\
hsa-miR-320b & 0.04421 & 0.98546 \\
hsa-miR-145-5p & 0.04545 & 0.98546 \\
hsa-miR-221-3p & 0.04598 & 0.98546 \\
hsa-miR-106a-5p & 0.04701 & 0.98546 \\
\hline
\end{tabular}

The table shows the 28 statistically significant miRNAs values with altered expressed levels among 100 miRNAs investigated during the observation time before correction for multiple testing and after correction $(n=8)$, sorted according to the lowest adjusted $p$-value.

The normalized expression (global mean method and adjusted for age and sex) levels $(\triangle \mathrm{Cp})$ of hsa-miR-17-5p, hsa-miR-30e-5p, hsa-miR-93-5p, and hsa-miR-423-3p declined within the first 12 months after diagnosis and subsequently increased from one to five years after diagnosis. By contrast, the levels of hsa-miR-10b-5p, hsa-miR-99a-5p, hsa-miR-125b-5p, and hsa-miR-497-5p increased during the first 12 months and subsequently decreased between one and five years after diagnosis. Five miRNAs (hsa-miR-10b-5p, hsa-miR-17-5p, hsa-miR-99a-5p, hsa-miR-125b-5p, and hsa-miR-497-5p) were expressed with a lower $\Delta \mathrm{Cp}$ value compared to the average of all samples at each time point, and the remaining three miRNAs (hsa-miR-30e-5p, hsa-miR-93-5p, and hsa-miR-423-3p) were more abundantly expressed compared to the average of all samples at all time-points (Figure 1 and Table S1).

\subsection{Identification of miRNA Target Genes}

The target genes for the eight candidate miRNAs were retrieved from two sources: miRTarBase [16] and TargetScan [17] using cyTargetLinker [18]. In total, 996 target genes for eight miRNAs as predicted by both tools were used for further analysis (Table S1). The pathway enrichment analysis was performed using PantherDB [19]. The pathway analysis revealed enrichment of various signaling pathways including epidermal growth factor (EGF), fibroblast growth factor (FGF), integrin, platelet-derived growth factor (PDGF), transforming growth factor (TGF) $\beta$, and apoptosis signaling pathways, as shown in Table 3. The detailed pathway analysis including the enriched target genes associated with each pathway are listed (Table S2). 
Table 3. Enriched pathways associated with the validated target genes of the eight miRNAs with altered expression levels during the first five years after diagnosis.

\begin{tabular}{|c|c|c|c|}
\hline Panther Pathways & Fold Enrichment & $p$-Value & miRNAs \\
\hline Angiogenesis & 3.37 & $8.25 \times 10^{-6}$ & $\begin{array}{l}\text { hsa-miR-10b-5p, hsa-miR-125b-5p, hsa-miR-17-5p, } \\
\text { hsa-miR-423-3p, hsa-miR-93-5p, hsa-miR-99a-5p }\end{array}$ \\
\hline $\begin{array}{l}\text { Gonadotropin-releasing hormone } \\
\text { receptor pathway }\end{array}$ & 2.9 & $1.77 \times 10^{-5}$ & $\begin{array}{l}\text { hsa-miR-17-5p, hsa-miR-125b-5p, hsa-miR-497-5p, } \\
\text { hsa-miR-423-3p, hsa-miR-10b-5p, hsa-miR-93-5p, } \\
\text { hsa-miR-30e-5p, hsa-miR-99a-5p }\end{array}$ \\
\hline TGF- $\beta$ signaling pathway & 4.08 & $7.21 \times 10^{-5}$ & $\begin{array}{l}\text { hsa-miR-10b-5p, hsa-miR-125b-5p, hsa-miR-17-5p, } \\
\text { hsa-miR-423-3p, hsa-miR-93-5p, hsa-miR-99a-5p }\end{array}$ \\
\hline CCKR signaling map & 3.11 & $1.07 \times 10^{-4}$ & $\begin{array}{l}\text { hsa-miR-17-5p, hsa-miR-125b-5p, hsa-miR-497-5p, } \\
\text { hsa-miR-93-5p, hsa-miR-99a-5p, hsa-miR-10b-5p, } \\
\text { hsa-miR-423-3p }\end{array}$ \\
\hline EGF receptor signaling pathway & 3.12 & $1.16 \times 10^{-3}$ & $\begin{array}{l}\text { hsa-miR-10b-5p, hsa-miR-125b-5p, hsa-miR-17-5p, } \\
\text { hsa-miR-423-3p, hsa-miR-497-5p, hsa-miR-93-5p, } \\
\text { hsa-miR-99a-5p }\end{array}$ \\
\hline PDGF signaling pathway & 2.91 & $3.16 \times 10^{-3}$ & $\begin{array}{l}\text { hsa-miR-10b-5p, hsa-miR-125b-5p, hsa-miR-17-5p, } \\
\text { hsa-miR-423-3p, hsa-miR-497-5p, hsa-miR-93-5p, } \\
\text { hsa-miR-99a-5p }\end{array}$ \\
\hline Apoptosis signaling pathway & 3.12 & $5.05 \times 10^{-3}$ & $\begin{array}{l}\text { hsa-miR-10b-5p, hsa-miR-125b-5p, hsa-miR-17-5p, } \\
\text { hsa-miR-30e-5p, hsa-miR-423-3p, hsa-miR-93-5p }\end{array}$ \\
\hline FGF signaling pathway & 3.05 & $6.90 \times 10^{-3}$ & $\begin{array}{l}\text { hsa-miR-10b-5p, hsa-miR-125b-5p, hsa-miR-17-5p, } \\
\text { hsa-miR-423-3p, hsa-miR-497-5p, hsa-miR-93-5p, } \\
\text { hsa-miR-99a-5p }\end{array}$ \\
\hline p53 pathway feedback loops 2 & 4.49 & $7.85 \times 10^{-3}$ & $\begin{array}{l}\text { hsa-miR-10b-5p, hsa-miR-125b-5p, hsa-miR-17-5p, } \\
\text { hsa-miR-423-3p, hsa-miR-93-5p, hsa-miR-99a-5p }\end{array}$ \\
\hline Ras Pathway & 3.66 & $1.34 \times 10^{-2}$ & $\begin{array}{l}\text { hsa-miR-10b-5p, hsa-miR-125b-5p, hsa-miR-17-5p, } \\
\text { hsa-miR-423-3p, hsa-miR-497-5p, hsa-miR-93-5p }\end{array}$ \\
\hline Integrin signalling pathway & 2.41 & $3.07 \times 10^{-2}$ & $\begin{array}{l}\text { hsa-miR-10b-5p, hsa-miR-125b-5p, hsa-miR-17-5p, } \\
\text { hsa-miR-423-3p, hsa-miR-497-5p, hsa-miR-93-5p, } \\
\text { hsa-miR-99a-5p }\end{array}$ \\
\hline
\end{tabular}

The enriched pathways are listed for the validated targets of the eight candidate miRNAs which changed in expression level over time. The fold enrichment and $p$-values were calculated using the binomial test. The $p$-values were adjusted for multiple correction using the Bonferroni method. TGF: transforming growth factor. CCKR: cholecystokinin receptors 1 and 2. EGF: epidermal growth factor. PDGF: platelet-derived growth factor. FGF: fibroblast growth factor. IGF: insulin-like growth factor.

\subsection{Partial remission and Immunological Status}

Using the new definition for PR with IDAA1c $\leq 9$ in the study population of 40 children and adolescents, 19 (51\%) children were in PR 3 months after diagnosis, and $4(12 \%)$ and $1(3 \%)$ were in PR after 12 and 60 months, respectively.

Of the five autoantibodies tested, seroconversion was most profound for ZnT8tripleAb during the study period. Insulin antibodies (IA) increased from 80 to 100\% during the first year after diagnosis (Table 1) and remained positive in all the children between three and twelve months in the study population and the study cohort. Seroconversion for IA was observed in twenty-two percent of the children five years after diagnosis (Table 1). The Spearman correlation analysis performed on the association of the eight candidate miRNAs and the anti-islet autoantibodies measurements showed that three of the candidate miRNAs were statistically significantly $(p<0.036)$ associated with the measured autoantibodies before correcting for multiple testing. Islet-cell autoantibodies (ICA) were negatively associated with hsa-miR-99a-5p at 3 months after diagnosis. At the same time point, ICA and IA-2A were negatively associated with hsa-miR-125b-5p. Finally, GADA65 was positively associated with hsa-miR-17-5p 6 months after diagnosis (Table 4). 
Table 4. Association between the eight candidate miRNAs and autoantibodies titers.

\begin{tabular}{cccccc}
\hline Months after Diagnosis & miRNA & Autoantibody & Spearman Correlation ( $\mathbf{r}_{\mathbf{s}}$ ) & $\begin{array}{c}\text { Unadjusted } \\
\boldsymbol{p} \text {-Value }\end{array}$ & $\begin{array}{c}\text { Adjusted } \\
\boldsymbol{p} \text {-Values }\end{array}$ \\
\hline 3 & hsa-miR-99a-5p & ICA & -0.35 & 0.036 & $\mathrm{~N} / \mathrm{S}$ \\
3 & hsa-miR-125b-5p & ICA & -0.36 & 0.030 & $\mathrm{~N} / \mathrm{S}$ \\
3 & hsa-miR-125b-5p & IA-2A & -0.35 & 0.032 & $\mathrm{~N} / \mathrm{S}$ \\
6 & hsa-miR-17-5p & GADA & 0.37 & 0.033 & $\mathrm{~N} / \mathrm{S}$ \\
\hline
\end{tabular}

The association between the eight candidate miRNAs and autoantibody titers measured at the five time points after diagnosis by Spearman correlation $\left(\mathrm{r}_{\mathrm{s}}\right)$. Only the statistically significant associations are shown $(p \leq 0.05)$. The significant values are before correcting for multiple testing. After correction, no significant association was seen (N/S). GAD65A: autoantibodies against antigen GAD. IA-2A: islet antigen 2. ICA: Islet-cell antibody. ZnT8: zinc transporter 8 (triple mix antibodies containing the isotypes glutamine, arginine, and tryptophan).

\subsection{Association of the Eight Candidate miRNAs to Cytokines}

The Spearman correlation analysis performed between the eight candidate miRNAs and 35 measured cytokines at 1,3,6, and 12 months after diagnosis showed, before correction for multiple testing, that hsa-miR-10b-5p correlated negatively with IL-21 $(r=-0.63, p=0.0051)$ at 3 months, whereas hsa-miR-17-5p at 1 month correlated positively with IL-21 $(r=0.62, p=0.0048)$. Likewise, hsa-miR-17-5p was positively associated with IL-10 and IL-4 at 1 month $(r=0.68, p=0.0015 ; r=0.70$, $p=0.0008$; respectively). Finally, hsa-miR-30e-5p correlated positively with IL-22 at 6 months $(r=0.71$, $p=0.0015)$.

\section{Discussion}

Aberrantly circulating miRNA levels in plasma, serum, and urine samples from children with T1D were assessed recently by Osipova et al. [20], and the number of studies exploring the prognostic potential of miRNAs in different settings is steadily increasing [21-23]. Our group recently showed, in the same cohort as the one used in present study, that six miRNAs predicted stimulated C-peptide, $\mathrm{HbA1c}$, and the insulin dose adjusted $\mathrm{HbA1c}$ (IDAA1c) the first year after disease onset [11]. In the present study, we identified miRNAs with expression levels that were influenced by disease progression, but not associated to the decline in $\beta$-cell function. In this prospective study, disease progression had a statistically significant effect on the expression levels of eight candidate miRNAs, irrespective of age and sex. Further bioinformatics evaluation of the validated targets of these miRNAs and the observed cytokine and pancreatic antibody associations support a possible immunological impact reflected by circulating miRNAs during the PR. However, cytokine profiles were only quantified in about half of the study participants in the Danish Remission Phase Cohort due to technical reasons. Thus, the study was under-powered for fully exploring these potential associations. The pathway-based analysis identified highly significant pathways associated with the target genes of eight miRNA candidates. The top highly significant pathways included angiogenesis, TGF- $\beta$, cholecystokinin receptor (CCKR), EGF, PDGF, apoptosis, FGF, integrin signaling, and the Ras pathway. STAT3, a potential target gene for hsa-miR-93-5p and hsa-miR-125b-5p, is associated with angiogenesis, the Ras pathway, EGF receptor signaling, CCKR signaling, and PDGF signaling pathways. MTOR (a serine/threonine protein kinase), which is a target gene for hsa-miR-99a-5p, is associated with the PDGF signaling pathway. MTOR is a central regulator of cellular metabolism, growth, and survival [24]. The activation of MTOR regulates various effector functions of CD4+ and CD8+ T cells, and MTOR inhibitors (i.e., rapamycin) are used as potential immunosuppressive therapeutics for organ transplants, metabolic diseases, and various autoimmune disorders [25]. Furthermore, MTOR has been demonstrated to be involved in the onset and progression of autoimmune disorders including diabetes [26]. PIK3CD, a target gene for hsa-miR-10b-5p is a known mediator of immune responses and is associated with several pathways including angiogenesis, apoptosis signaling, EGF receptor signaling, PDGF signaling, FGF signaling, integrin signaling, and the Ras pathway.

One of the strengths of this study was the phenotypically thorough characterization of the study participants who were followed with repeated measurements initially after diagnosis and 
during the first five years thereafter. The present study also provides data from pathway analysis together with cytokine and pancreatic antibody variations associated with miRNAs during PR to suggest that miRNAs regulating genes that control immune recognition could be involved in the autoimmune processes during the initial progression of the disease. In our previous study [11], six miRNAs analyzed in the same population could predict residual $\beta$-cell function and glycemic control (SCP, $\mathrm{HbA1c}$, and IDAA1c) in children with new onset T1D the first five years after diagnosis. Accumulating evidence supports that miRNAs play a crucial role in the regulation of pancreatic $\beta$-cell function under normal and pathophysiological conditions, such as insulin biosynthesis, insulin exocytosis, and $\beta$-cell expansion, and may have clinical significance in diabetes [11,27]. The current study further provides data, in the same study population, on different miRNAs, which change expression levels during the first five years after diagnosis and have a possible involvement in immunological pathways. Three pancreatic autoantibodies, ICA, IA-2A, and GADA65, and four cytokines, IL-4, IL-10, IL-21, and IL-22, were associated with the miRNAs at different time points. Interestingly, hsa-miR-17-5p correlated with interleukin-4 and interleukin-10 levels, which have anti-inflammatory effects and dampens the autoimmune process. However, these stratifications were under-powered due to limited sample sizes and the limited number of study participants who were followed five years after diabetes diagnosis. This decreased the statistical power to conclude on the potential role of the eight candidate miRNAs during the PR. The observed variability of the candidate miRNAs need further validation in larger cohorts, and their immunological function in autoimmune diabetes also needs to be elucidated in further detail.

In conclusion, this study identified eight circulating miRNAs that changed in expression levels, irrespective of age and sex, during the first five years after diabetes diagnosis, which have the potential to be used as biomarkers for the immunological progression and definition of the optimal state for immune intervention during the initial phase of T1D.

\section{Materials and Methods}

\subsection{Study Population and Samples}

The Danish Remission Phase Cohort comprised 129 children (66 boys) with T1D. The study was a longitudinal multicenter investigation conducted in four pediatric out-patient clinics with enrolment during the years 2004 to 2005. Plasma samples were drawn on fasting state after a meal stimulation test at $1,3,6,12$, and 60 months after diagnosis and stored in a bio-bank at $-80^{\circ} \mathrm{C}$ until further use. Diabetes diagnosis was classified according to the World Health Organization criteria [28]. The reasons for drop-outs from the cohort or loss of follow up at the 5-year visit were either adolescents transferred to adult clinics, changed residences, or unwillingness to participate. The exclusion criteria were suspicions of other types of diabetes (maturity-onset diabetes of the young (MODY), type 2 diabetes, or secondary diabetes) and / or if patients were initially treated outside the center for more than five days. The 40 participants who completed the follow-up at five years diabetes duration were slightly younger with a lower body mass index (BMI) and had lower postprandial C-peptide levels 12 months after diagnosis compared to the full cohort, but they were similar in terms of sex distribution, $\mathrm{HbA1c}$, and hormone levels. The study was performed according to the criteria of the Helsinki II Declaration and was approved by the Danish National Committee on Biomedical Research Ethics (Journal number: H-KA-04010-m). Older children gave their assent and all parents or guardians gave written informed consent.

\subsection{Partial Remission (PR) Phase}

Different definitions for the PR phase have been suggested. Previously, it was defined as $\mathrm{HbA1c}$ near or within the normal range and a daily insulin dose requirement of $<0.5$ units $/ \mathrm{kg} /$ day [29]. A new definition combining both values has recently been proposed: insulin dose-adjusted $\mathrm{HbA} 1 \mathrm{c}$, 
and IDAA1c, which suggests that a value $\leq 9$ indicates partial remission [30,31]. This definition has been recommended by the International Society for Pediatric and Adolescent Diabetes (ISPAD) [32].

\subsection{Pancreatic Anti-Islet Autoantibodies}

Islet autoantibody measurements were measured in serum samples from all included children in the Danish Remission Phase Cohort. Titers of the anti-islet autoantibodies (insulin antibodies (IA), insulinoma associated antigen-2 autoantibody (IA-2A), autoantibodies against glutamic acid decarboxylase (GAD65A), and islet-cell autoantibodies (ICA)) were analyzed at 1, 3, 6, 12, and 60 months after diagnosis according to the methods described earlier [33]. The zinc transporter 8 variant containing all three isotypes arginine, tryptophan, and glutamine (ZnT8RWQ or ZnT8tripleAb) were analyzed by a so called triple mix radioligand binding assay (RBA) as previously described $[34,35]$ at the time points $1,3,6$, and 12 months after diagnosis. Another method was used for analyzing the "triple mix" ZnT8 autoantibodies (ZnT8tripleAb) 60 months after diagnosis [36].

The cut-off values for anti-islet autoantibody positivity were 5.36 relative units (RU) for GAD65A, 0.77 RU for IA-2A, 2.5 Juvenile Diabetes Foundation units (JDFU) for ICA, and 2.80 RU for IA. The cut-off limit for the triple mix assay, used the first year after diagnosis, was $58 \mathrm{U} / \mathrm{mL}$, and for the assay by Salonen et al. [36] used five years after diagnosis, the cut-off value was set to $0.61 \mathrm{RU}$.

\subsection{Cytokine Measurements}

Cytokine status was measured for half of the recruited study participants in the Danish Remission Phase Cohort at 1, 3, 6, and 12 months after diagnosis. Cytokine measurements were performed using an in-house-developed and -validated multiplex immunoassay (Laboratory of Translational Immunology, University Medical Center, Utrecht, The Netherlands) based on Luminex technology (xMAP, Luminex, Austin TX, USA) [37].

\section{5. miRNA Expression Profiling and Normalization Method}

miRNAs were quantified from a total of 182 plasma samples drawn from the subgroup of 40 children and adolescents who participated in the 5-year visit. RNA extraction was performed using the miRCURYTM RNA Isolation Kit-Biofluids and RT-qPCR was performed with ExiLENT SYBR ${ }^{\circledR}$ Green Master Mix according to the manufacturer's instructions (Exiqon A/S, Vedbaek, Denmark). All RT-qPCR assays were performed in a LightCycler $4800^{\circledR}$ Real-Time PCR System in 384 well plates (Roche, Hvidovre, Denmark). Quality control was done on a subset of samples $(n=10)$ with synthetic spike-in RNAs as a quality control of the RNA isolation and cDNA synthesis. A detailed description of the RNA isolation and quality control procedure is outlined in [9]. Assessment of hemolysis was evaluated by comparing the levels of hsa-miR-451a (miRNA highly expressed in erythrocytes) with hsa-miR-23a-3p (stable in plasma/serum and not affected by hemolysis) as described by Blondal et al. [38].

Each plasma sample was run on a selected assay containing a panel of 179 predefined human serum/plasma miRNAs (V3; Exiqon), including miRNAs known to be involved in immunological and metabolic diseases. The $100 \mathrm{miRNAs}$ with complete data that were quantified in all samples in the miRNA expression profiling were employed for the normalization of data using the global mean method, since this approach has been shown to be the most stable normalizer according to Mestdagh et al. [39]. The formula used to calculate the normalized $\mathrm{Cp}$ values was:

$$
\Delta \mathrm{Cp}=\text { average } \mathrm{Cp} \text { (all samples) - assay } \mathrm{Cp} \text { (sample) }
$$

\subsection{Validation of Target Genes and Pathway Analysis}

The validated and predicted target genes for the eight candidate miRNAs found in this study were retrieved from two sources: miRTarBase [16] (experimentally verified miRNA interactions retrieved from automated text mining in PubMed) and TargetScan [17] (predicted miRNA interactions) using 
the cyTargetLinker app in CytoScape [18]. The pathway-based enrichment analysis was performed using PantherDB [19]. The $p$-values were calculated using the binomial test and adjusted for multiple corrections using the Bonferroni method.

\subsection{Statistical Analysis}

All statistical analyses were performed using SAS version 9.4 (SAS Institute, Cary, NC, USA). The miRNA levels with complete data were included for analysis and were managed on a base-2 logarithmic scale $(\log 2)$. The influence of diabetes duration on circulating levels of miRNAs were analyzed by a linear mixed model for repeated measurements including the covariates for sex and age at the visit (to account for the potential age development) and with an unstructured covariance matrix. $p$-Values for the differentially expressed miRNAs associated with disease duration were further adjusted according to the Hochberg approach $(p<0.05)$. For antibodies and cytokines, the Spearman correlation analysis used the measured values and included values below the detection limit as a value below all others; it was performed to determine the relationship between variables and was considered significant with a corresponding $p \leq 0.05$.

Supplementary Materials: The following are available online at http:/ / www.mdpi.com/2311-553X/4/4/35/s1, Table S1: $\Delta \mathrm{Cp}$ values of 8 miRNAs at different time-points and Table S2: Pathway-based enrichment analysis using PantherDB.

Author Contributions: N.S., L.B.N., H.B.M., and F.P. designed the study; P.H. performed the statistical analysis; A.H.M. and S.K. performed the bioinformatics analyses; S.F. was involved in collection and characterization of the cohort; all authors were involved in drafting the manuscript, approving the final draft, and agreed to be accountable for the work; all authors read and approved the final manuscript.

Funding: This research was conducted with support from the Capital Region of Denmark's Research Fund for Health Science, European Foundation for the Study of Diabetes (EFSD-Novo Nordisk-JDRF), Beckett Foundation, and Savværksejer Jeppe Juhl og hustru Ovita Juhls mindelegat. The study sponsor was not involved in the design of the study, the collection, analysis and interpretation of the data, writing of the report, or the decision to submit the report for publication.

Acknowledgments: The authors of this paper sincerely wish to thank the following investigators for their substantial contributions in conducting this study: S. Pörksen (Pediatric Department, Roskilde Hospital, Roskilde, Denmark) for his contribution in outlining the protocol and collection of data for the Danish Remission Phase Cohort; and M.L.M. Andersen (Psychiatric Department for Children and Adolescents, Glostrup Hospital, Glostrup, Denmark) for recruiting patients for the follow-up study 5 years after diagnosis. Furthermore, we wish to thank L. Hansen (CPH-DIRECT, Department of Paediatrics E, Herlev and Gentofte Hospital, Herlev, Denmark) for valuable contributions to critically revising the manuscript. Some of the data were presented as an abstract and poster at the 52nd Annual EASD Meeting in 2016, Munich, Germany.

Conflicts of Interest: The authors declare no conflict of interest.

\section{References}

1. Redondo, M.J.; Yu, L.; Hawa, M.; Mackenzie, T.; Pyke, D.A.; Eisenbarth, G.S.; Leslie, R.D. Heterogeneity of type I diabetes: Analysis of monozygotic twins in Great Britain and the United States. Diabetologia 2001, 44, 354-362. [CrossRef] [PubMed]

2. Pociot, F.; Lernmark, Å. Genetic risk factors for type 1 diabetes. Lancet 2016, 387, 2331-2339. [CrossRef]

3. Knip, M.; Simell, O. Environmental triggers of type 1 diabetes. Cold Spring Harb. Perspect. Med. 2012, 2, a007690. [CrossRef] [PubMed]

4. Stefan, M.; Zhang, W.; Concepcion, E.; Yi, Z.; Tomer, Y. DNA methylation profiles in type 1 diabetes twins point to strong epigenetic effects on etiology. J. Autoimmun. 2014, 50, 33-37. [CrossRef] [PubMed]

5. Dang, M.N.; Buzzetti, R.; Pozzilli, P. Epigenetics in autoimmune diseases with focus on type 1 diabetes. Diabetes Metab. Res. Rev. 2013, 29, 8-18. [CrossRef] [PubMed]

6. Blackard, J.T.; Kong, L.; Lombardi, A.; Homann, D.; Hammerstad, S.S.; Tomer, Y. A preliminary analysis of hepatitis C virus in pancreatic islet cells. Virol. J. 2017, 14, 237. [CrossRef] [PubMed]

7. Lombardi, A.; Tsomos, E.; Hammerstad, S.S.; Tomer, Y. Interferon- $\alpha$ : The key trigger of type 1 diabetes. J. Autoimmun. 2018, 94, 7-15. [CrossRef] [PubMed] 
8. Wang, J.; Chen, J.; Sen, S. MicroRNA as biomarkers and diagnostics. J. Cell. Physiol. 2016, 231, 25-30. [CrossRef] [PubMed]

9. Guay, C.; Regazzi, R. Circulating microRNAs as novel biomarkers for diabetes mellitus. Nat. Rev. Endocrinol. 2013, 9, 513-521. [CrossRef] [PubMed]

10. Kantharidis, P.; Wang, B.; Carew, R.M.; Lan, H.Y. Diabetes complications: The microRNA perspective. Diabetes 2011, 60, 1832-1837. [CrossRef] [PubMed]

11. Samandari, N.; Mirza, A.H.; Nielsen, L.B.; Kaur, S.; Hougaard, P.; Fredheim, S.; Mortensen, H.B.; Pociot, F. Circulating microRNA levels predict residual $\beta$-cell function and glycaemic control in children with type 1 diabetes mellitus. Diabetologia. 2017, 60, 354-363. [CrossRef] [PubMed]

12. Ventriglia, G.; Nigi, L.; Sebastiani, G.; Dotta, F. MicroRNAs: Novel players in the dialogue between pancreatic islets and immune system in autoimmune diabetes. Biomed. Res. Int. 2015, 2015, 749734. [CrossRef] [PubMed]

13. Miao, F.; Smith, D.D.; Zhang, L.; Min, A.; Feng, W.; Natarajan, R. Lymphocytes from patients with type 1 diabetes display a distinct profile of chromatin histone $\mathrm{H} 3$ lysine 9 dimethylation: An epigenetic study in diabetes. Diabetes 2008, 57, 3189-3198. [CrossRef] [PubMed]

14. Rakyan, V.K.; Beyan, H.; Down, T.A.; Hawa, M.I.; Maslau, S.; Aden, D.; Daunay, A.; Busato, F.; Mein, C.A.; Manfras, B. Identification of type 1 diabetes-associated DNA methylation variable positions that precede disease diagnosis. PLoS Genet. 2011, 7, e1002300. [CrossRef] [PubMed]

15. Abdul-Rasoul, M.; Habib, H.; Al-Khouly, M. "The honeymoon phase" in children with type 1 diabetes mellitus: Frequency, duration, and influential factors. Pediatr. Diabetes. 2006, 7, 101-107. [CrossRef] [PubMed]

16. Chou, C.H.; Shrestha, S.; Yang, C.D.; Chang, N.W.; Lin, Y.L.; Liao, K.W.; Huang, W.C.; Sun, T.H.; Tu, S.J.; Lee, W.H.; et al. miRTarBase update 2018: a resource for experimentally validated microRNA-target interactions. Nucleic Acids Res. 2018, 46, D296-D302. [CrossRef] [PubMed]

17. Agarwal, V.; Bell, G.W.; Nam, J.W.; Bartel, D.P. Predicting effective microRNA target sites in mammalian mRNAs. eLife 2015, 4, e05005. [CrossRef] [PubMed]

18. Kutmon, M.; Kelder, T.; Mandaviya, P.; Evelo, C.T.A.; Coort, S.L. CyTargetLinker: A cytoscape app to integrate regulatory interactions in network analysis. PLoS ONE 2013, 8, e82160. [CrossRef] [PubMed]

19. Mi, H.; Poudel, S.; Muruganujan, A.; Casagrande, J.T.; Thomas, P.D. PANTHER version 10: Expanded protein families and functions, and analysis tools. Nucl. Acids Res. 2016, 44, D336-D342. [CrossRef] [PubMed]

20. Osipova, J.; Fischer, D.-C.; Dangwal, S.; Volkmann, I.; Widera, C.; Schwarz, K.; Lorenzen, J.M.; Schreiver, C.; Jacoby, U.; Heimhalt, M. Diabetes-associated microRNAs in pediatric patients with type 1 diabetes mellitus: A cross-sectional cohort study. J. Clin. Endocrinol. MeTable 2014, 99, E1661-E1665. [CrossRef] [PubMed]

21. Nielsen, L.B.; Wang, C.; Sørensen, K.; Bang-Berthelsen, C.H.; Hansen, L.; Andersen, M.-L.M.; Hougaard, P.; Juul, A.; Zhang, C.-Y.; Pociot, F. Circulating levels of microRNA from children with newly diagnosed type 1 diabetes and healthy controls: Evidence that miR-25 associates to residual $\beta$-cell function and glycaemic control during disease progression. Exp. Diabetes Res. 2012, 2012, 896362. [CrossRef] [PubMed]

22. Nabih, E.S.; Andrawes, N.G. The association between circulating levels of miRNA-181a and pancreatic $\beta$-cells dysfunction via SMAD7 in type 1 diabetic children and adolescents. J. Clin. Lab. Anal. 2016, 30, 727-731. [CrossRef] [PubMed]

23. Marchand, L.; Jalabert, A.; Meugnier, E.; Van den Hende, K.; Fabien, N.; Nicolino, M.; Madec, A.-M.; Thivolet, C.; Rome, S. miRNA-375 a sensor of glucotoxicity is altered in the serum of children with newly diagnosed type 1 diabetes. J. Diabetes Res. 2016, 2016, 1869082. [CrossRef] [PubMed]

24. Laplante, M.; Sabatini, D.M. mTOR signaling in growth control and disease. Cell 2012, 149, $274-293$. [CrossRef] [PubMed]

25. Perl, A. mTOR activation is a biomarker and a central pathway to autoimmune disorders, cancer, obesity, and aging. Ann. N. Y. Acad. Sci. 2015, 1346, 33-44. [CrossRef] [PubMed]

26. Zoncu, R.; Efeyan, A.; Sabatini, D.M. mTOR: From growth signal integration to cancer, diabetes and ageing. Nat. Rev. Mol. Cell Biol. 2011, 12, 21-35. [CrossRef] [PubMed]

27. Özcan, S. microRNAs in pancreatic $\beta$-Cell physiology. Adv. Exp. Med. Biol. 2015, 887, 101-117. [CrossRef] [PubMed]

28. Genuth, S.; Alberti, K.G.M.M.; Bennett, P.; Buse, J.; Defronzo, R.; Kahn, R.; Kitzmiller, J.; Knowler, W.C.; Lebovitz, H.; Lernmark, A. Committee on the diagnosis and classification of diabetes mellitus follow-up report on the diagnosis of diabetes mellitus. Diabetes Care 2003, 26, 3160-3167. [PubMed] 
29. Lombardo, F.; Valenzise, M.; Wasniewska, M.; Messina, M.F.; Ruggeri, C.; Arrigo, T.; De Luca, F. Two-year prospective evaluation of the factors affecting honeymoon frequency and duration in children with insulin dependent diabetes mellitus: The key-role of age at diagnosis. Diabetes Nutr. MeTable 2002, 15, 246-251.

30. Mortensen, H.B.; Hougaard, P.; Swift, P.; Hansen, L.; Holl, R.W.; Hoey, H.; Bjoerndalen, H.; de Beaufort, C.; Chiarelli, F.; Danne, T. Study group on childhood diabetes new definition for the partial remission period in children and adolescents with type 1 diabetes. Diabetes Care 2009, 32, 1384-1390. [CrossRef] [PubMed]

31. Max Andersen, M.L.C.; Hougaard, P.; Pörksen, S.; Nielsen, L.B.; Fredheim, S.; Svensson, J.; Thomsen, J.; Vikre-Jørgensen, J.; Hertel, T.; Petersen, J.S. Partial remission definition: Validation based on the insulin dose-adjusted HbA1c (IDAA1C) in 129 Danish children with new-onset type 1 diabetes. Pediatr. Diabetes 2014, 15, 469-476. [CrossRef] [PubMed]

32. Couper, J.J.; Haller, M.J.; Ziegler, A.-G.; Knip, M.; Ludvigsson, J.; Craig, M.E.; International Society for Pediatric and Adolescent Diabetes. ISPAD clinical practice consensus guidelines 2014. Phases of type 1 diabetes in children and adolescents. Pediatr. Diabetes. 2014, 15 (Suppl. 20), 18-25. [CrossRef]

33. Andersen, M.L.M.; Vaziri-Sani, F.; Delli, A.; Pörksen, S.; Jacobssen, E.; Thomsen, J.; Svensson, J.; Steen Petersen, J.; Hansen, L.; Lernmark, A. Association between autoantibodies to the Arginine variant of the Zinc transporter 8 (ZnT8) and stimulated C-peptide levels in Danish children and adolescents with newly diagnosed type 1 diabetes. Pediatr. Diabetes 2012, 13, 454-462. [CrossRef] [PubMed]

34. Vaziri-Sani, F.; Delli, A.J.; Elding-Larsson, H.; Lindblad, B.; Carlsson, A.; Forsander, G.; Ivarsson, S.A.; Ludvigsson, J.; Marcus, C.; Lernmark, A. A novel triple mix radiobinding assay for the three ZnT8 (ZnT8-RWQ) autoantibody variants in children with newly diagnosed diabetes. J. Immunol. Methods 2011, 371, 25-37. [CrossRef] [PubMed]

35. Vaziri-Sani, F.; Oak, S.; Radtke, J.; Lernmark, K.; Lynch, K.; Agardh, C.-D.; Cilio, C.M.; Lethagen, A.L.; Ortqvist, E.; Landin-Olsson, M. ZnT8 autoantibody titers in type 1 diabetes patients decline rapidly after clinical onset. Autoimmunity 2010, 43, 598-606. [CrossRef] [PubMed]

36. Salonen, K.M.; Ryhänen, S.; Härkönen, T.; Ilonen, J.; Knip, M. Finnish pediatric diabetes register autoantibodies against zinc transporter 8 are related to age, metabolic state and HLA DR genotype in children with newly diagnosed type 1 diabetes. Diabetes Metab. Res. Rev. 2013, 29, 646-654. [CrossRef] [PubMed]

37. De Jager, W.; Prakken, B.J.; Bijlsma, J.W.J.; Kuis, W.; Rijkers, G.T. Improved multiplex immunoassay performance in human plasma and synovial fluid following removal of interfering heterophilic antibodies. J. Immunol. Methods 2005, 300, 124-135. [CrossRef] [PubMed]

38. Blondal, T.; Jensby Nielsen, S.; Baker, A.; Andreasen, D.; Mouritzen, P.; Wrang Teilum, M.; Dahlsveen, I.K. Assessing sample and miRNA profile quality in serum and plasma or other biofluids. Methods 2013, 59, S1-S6. [CrossRef] [PubMed]

39. Mestdagh, P.; Van Vlierberghe, P.; De Weer, A.; Muth, D.; Westermann, F.; Speleman, F.; Vandesompele, J. A novel and universal method for microRNA RT-qPCR data normalization. Gen. Biol. 2009, 10, R64. [CrossRef] [PubMed]

(C) 2018 by the authors. Licensee MDPI, Basel, Switzerland. This article is an open access article distributed under the terms and conditions of the Creative Commons Attribution (CC BY) license (http://creativecommons.org/licenses/by/4.0/). 